Enfermagem Brasil 2016;15(3):123-30

\title{
ARTIGO ORIGINAL \\ Perfil epidemiológico e social de mulheres internadas em um hospital universitário de Minas Gerais
}

Fernanda Canela Prates*, Simone Guimarães Teixeira Souto, M.Sc. ${ }^{*}$, Ana Paula Ferreira Holzmann, M.Sc. ${ }^{* *}$, Maria da Salette Mendonça ${ }^{* *}$

*Enfermeira, Especialista na modalidade Residência em Saúde da Mulher pela UNIMONTES, Docente do curso técnico em enfermagem do Colégio Indyu, ${ }^{* *}$ Enfermeira, Docente do departamento de enfermagem da Unimontes, ${ }^{\star * \star}$ Enfermeira efetiva do Hospital Universitário Clemente de Faria

Recebido em 16 de março de 2015; aceito em 12 de junho de 2015.

Endereço para correspondência: Fernanda Canela Prates, Rua Antenor Leite, 12A, Vila Guilhermina, 39400-491 Montes Claros MG, E-mail: fernanda_canela@hotmail.com, Simone Guimarães Teixeira Souto: simonegts28@yahoo.com.br, Ana Paula Ferreira Holzmann: apaulah@uol.com.br, Maria da Salette Mendonça: mariadasalettem@yahoo.com.br

\section{Resumo}

A morbidade hospitalar na população feminina em Minas Gerais foi de 22.616 e em Montes Claros, 902, conforme o Departamento de Informática do Sistema Único de Saúde - DATASUS do ano 2012. Este estudo objetivou conhecer o perfil epidemiológico e social das mulheres atendidas e internadas em um hospital universitário. Trata-se de uma pesquisa quantitativa de abordagem descritiva com objetivo exploratório. A análise dos dados foi construída pelo programa Microsoft Office Excel 2007. A pesquisa foi realizada no Hospital Universitário Clemente de Faria em Montes Claros/MG com 30 prontuários de mulheres internadas na clínica médica. Pode-se concluir que a maioria das mulheres eram adultas e solteiras; a principal patologia encontrada foi a pneumonia, embora houvesse maior ocorrência das doenças do sistema gastrintestinal; e a comorbidade mais apresentada foi a hipertensão arterial. Os achados podem contribuir para o aperfeiçoamento do cuidado e melhoria na qualidade de atendimento específico.

Palavras-chave: morbidade, saúde da mulher, hospitalização, epidemiologia.

\section{Abstract}

\section{Epidemiological and social profile of women admitted to university hospital in Minas Gerais}

The hospital morbidity in the female population in the state of Minas Gerais was 22,616 and in Montes Claros, 902, according to the Informatics Department of Brazilian Public Health System - DATASUS for the year 2012. This study investigated the epidemiological and social profile of women hospitalized in a university hospital. It is a descriptive study with quantitative approach and exploratory aim. Data was analyzed using Microsoft Office Excel 2007. The research was performed at the University Hospital Clement Faria in Montes Claros/MG with 30 medical records of women hospitalized in a medical center. It can be concluded that many of the women were adults and unmarried; pneumonia was the most common pathology, with an higher incidence of gastrointestinal diseases; and hypertension was the most common comorbidity. The findings can contribute to the improvement of care and improving the quality of care.

Key-words: morbidity, women's health, hospitalization, epidemiology.

\section{Resumen \\ Perfil epidemiológico y social de mujeres ingresadas en un hospital universitario en Minas Gerais}

La morbilidad hospitalaria en la población femenina en Minas Gerais se situó en 22.616 y en Montes Claros, 902, según el Departamento de Informática del Sistema Único de Salud DATASUS/2012. Este estudio tuvo como objetivo conocer elperfil epidemiológico y social de mujeres tratadas e ingresadas en un hospital universitario. Se trata de una investigación cuantitativa de enfoque descriptivo con objetivo exploratorio. El análisis de datos fue construido por el programa Microsoft Office Excel 2007. La investigación se llevó a cabo en el Hospital 
Universitario Clemente de Faria en Montes Claros/MG con 30 registros de mujeres ingresadas en la clínica médica. Se puede concluir que la mayoría de las mujeres eran adultas y solteras; la principal patología encontrada fue la neumonía, aunque las enfermedades del sistema gastrointestinal aparecieron en mayor cantidad; y la comorbilidad principal fue la hipertensión arterial. Los hallazgos pueden contribuir a la mejora de los cuidados y de la calidad de la atención especializada.

Palabras-clave: morbilidad, salud de la mujer, hospitalización, epidemiología.

Introdução

O Censo Demográfico de 2010 apresentou uma população de 190.755 .799 habitantes no Brasil. Deste total, $51 \%$ são mulheres e $49 \%$ são homens, o número de mulheres supera em 3.941.819. A região Sudeste é a que possui o maior número de mulheres [1].

Minas Gerais é o segundo estado mais populoso do país, com o total de 19.597 .330 pessoas, das quais 9.955.452 são mulheres. No município de Montes Claros residem 361.915 habitantes, destes, 185.052 são mulheres [1]. Conforme o Departamento de Informática do Sistema Único de Saúde - DATASUS 2012, a morbidade hospitalar na população feminina em Minas Gerais é de 22.616 e em Montes Claros, 902 [2].

A morbidade da população permite avaliar os riscos de adoecer à que as pessoas estão submetidas, orientam investigações sobre os determinantes das doenças e apontam para intervenções necessárias. Uma grande vantagem da medida de morbidade é sua sensibilidade as mudanças a curto prazo [3].

Para bem receber a população nos estabelecimentos de saúde, o Ministério da Saúde atualmente tem buscado padronizar o processo de acolhimento, com classificação de risco por meio da adoção de medidas que uniformizem esse processo em todo o território nacional. Após a classificação de risco e avaliação médica, é definida a conduta e destino do usuário: alta externa (domicílio/referência) ou interna (observação/internação) [4].

Existindo uma padronização no acolhimento e direcionamento do usuário, a efetividade dos motivos de hospitalizações viabiliza as internações com acurácia. Este estudo justifica-se com base na escassez de publicações relativa à morbidade das mulheres. Entende-se que os resultados desta pesquisa contribuirão para conhecer os principais motivos que levam as mulheres a procurar assistência hospitalar, consequentemente lança-se o desafio da qualificação de profissionais para este público.

Sabendo que as causas de internação hospitalar sofrem variações intensas de acordo com o perfil epidemiológico da população estudada [5], o objetivo geral deste estudo foi conhecer o perfil epidemiológico e social das mulheres atendidas e internadas em um hospital universitário. Deste, elaborou-se os seguintes objetivos específicos: identificar os Códigos Internacionais das Doenças (CID) dos atendimentos, analisar as principais causas de internações hospitalares de mulheres e investigar a existência de comorbidades, associando às doenças prevalentes nas mulheres.

Material e métodos

Trata-se de uma pesquisa quantitativa de abordagem descritiva com objetivo exploratório. Procedeu-se tecnicamente de maneira documental transversal.

A pesquisa descritiva tem o objetivo de descrever fatos de uma determinada realidade [6]; no caso deste estudo, é a situação de saúde das mulheres atendidas e internadas no hospital referido, acrescida pela pesquisa exploratória que visa a uma primeira aproximação do pesquisador com o tema, para torná-lo mais familiarizado com os fatos e fenômenos relacionados ao problema a ser estudado [7].

Quanto ao procedimento técnico, é uma pesquisa documental por ter o levantamento de dados como base. Por fim, será desenvolvida de maneira transversal, ou seja, a pesquisa realiza-se em um curto período de tempo, em um determinado momento [7].

\section{Espaço de investigação}

A pesquisa foi realizada em um hospital de Montes Claros, no norte de Minas Gerais. É um hospital eminentemente público que dedica $100 \%$ dos seus leitos ao Sistema Único de 
Saúde (SUS). Possui 171 leitos hospitalares e 10 leitos de internação domiciliar. Sua abrangência vai desde o Norte de Minas, Vale do Jequitinhonha e Mucuri, Noroeste de Minas até o Sul da Bahia.

A amostra do estudo constituiu-se de prontuários das mulheres que procuraram assistência à saúde no pronto socorro do hospital e foram internadas na clínica médica, sendo excluídos da amostra aqueles que tinham queixa gineco-obstétrica, crianças e adolescentes.

\section{Levantamento e análise de dados}

A identificação de fontes para a coleta de dados é o primeiro passo para que se inicie o desenvolvimento da pesquisa sobre o objeto estudado [8].

Visando alcançar o objetivo da pesquisa utilizou-se como instrumento um formulário estruturado.

A utilização da pesquisa documental deu-se através dos prontuários clínicos de internação, para obtenção de dados referentes aos sujeitos em estudo, sendo transcritas para um formulário estruturado.

Etapas da pesquisa

O primeiro procedimento para obtenção de dados foi a consulta nos prontuários clínicos da clínica médica, analisados diariamente por um período de trinta dias, a fim de perceber as variáveis existentes no decorrer do mês.

A coleta de dados aconteceu durante o mês de dezembro de 2014, neste momento as informações inerentes à pesquisa foram descritas em formulário próprio para melhor tratamento dos dados.

Após a coleta de dados, iniciou-se a organização e a análise do material. Nesta fase torna-se evidente a "hesitante alquimia" do pesquisador, pois é o momento em que transforma dados brutos em descobertas finais [9]. Neste estudo, a análise dos dados foi construída pelo programa Microsoft Office Excel 2007 para apresentação dos dados coletados buscando confirmar ou não os pressupostos da pesquisa.

Os resultados e as discussões foram descritas por variáveis a serem exploradas em consonância com os objetivos da pesquisa. São elas: 1) Perfil das mulheres internadas. 2) Descrição dos CID e doenças prevalentes nas mulheres. 3) Comorbidades apresentadas e fatores associados à internação das mulheres.

\section{Aspectos éticos}

Em concordância com a Resolução ํo 466 de 12 de dezembro de 2012 que busca atender aos princípios da bioética, a instituição assinou o Termo de Concordância da Instituição para aceite da pesquisa, autorizando a coleta dos dados, bem como a divulgação da pesquisa e de seus resultados.

A pesquisa foi executada após ter sido aprovada pelo Comitê de Ética em Pesquisa (CEP) da Universidade Estadual de Montes Claros - Unimontes com o parecer de número 537.309.

\section{Resultados e discussão}

A amostra da pesquisa compreendeu 30 prontuários de mulheres que estiveram internadas na clínica médica do hospital, durante o mês de dezembro de 2014, tanto em tratamento de patologias crônicas quanto eventos agudos.

\section{Perfil das mulheres internadas}

A taxa de internação hospitalar está associada a fatores como idade, gênero, renda familiar e escolaridade [5]. As figuras a seguir mostram o perfil social - faixa etária, estado civil, etnia e cidade - das participantes do estudo. 
Figura 1 - Distribuição da idade.

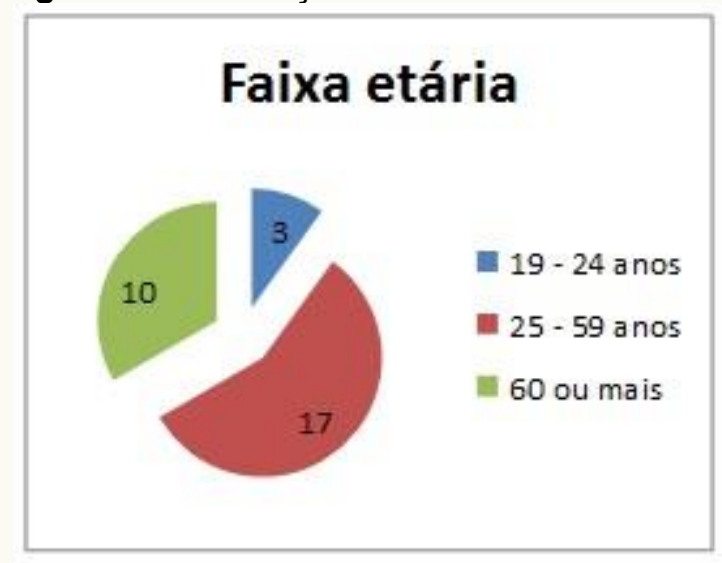

Figura 2 - Estado civil das participantes.

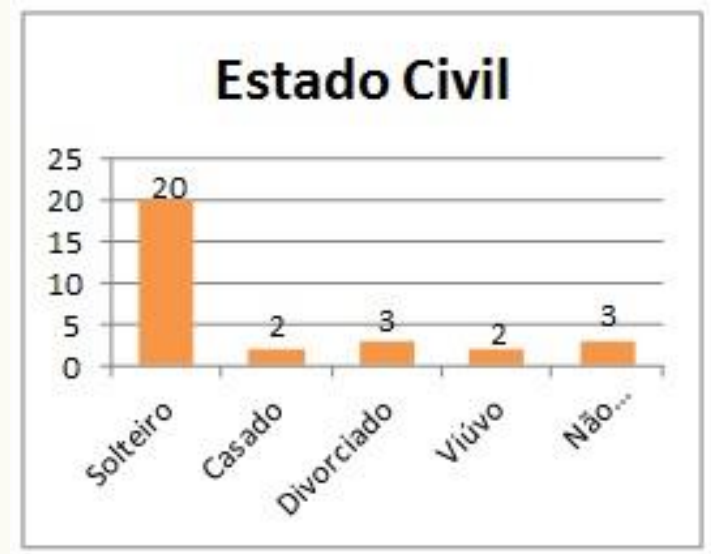

A idade das pacientes variou de 19 a 81 anos. Foram divididas em faixas etárias preconizadas pela Organização Mundial de Saúde, sendo a juventude de 19 a 24 anos, idade adulta de 25 a 59 anos, e a pessoa idosa com 60 anos ou mais. Na amostra $10 \%$ (3) equivale a mulheres jovens, $56,6 \%$ (17) adultas e $33,4 \%$ (10) idosas.

Conforme estudo realizado com adultos em um município da Amazônia Legal, a maior frequência de internação hospitalar foi para a faixa etária de 18 a 39 anos $(51,6 \%)$ [11]. Em outro estudo realizado no Rio Grande do Sul $57,2 \%$ das mulheres internadas estavam na faixa etária de 40 a 69 anos [10].

O estado civil foi informado no momento da internação. De $100 \%$ da amostra, $66,6 \%$ (20) eram solteiras, $6,7 \%$ (2) casadas, $10 \%$ (3) divorciadas, 6,7\% (2) viúvas e 10\% (3) não informaram, conforme apresenta a Figura 2. 
Figura 3 - Raça autodeclarada.

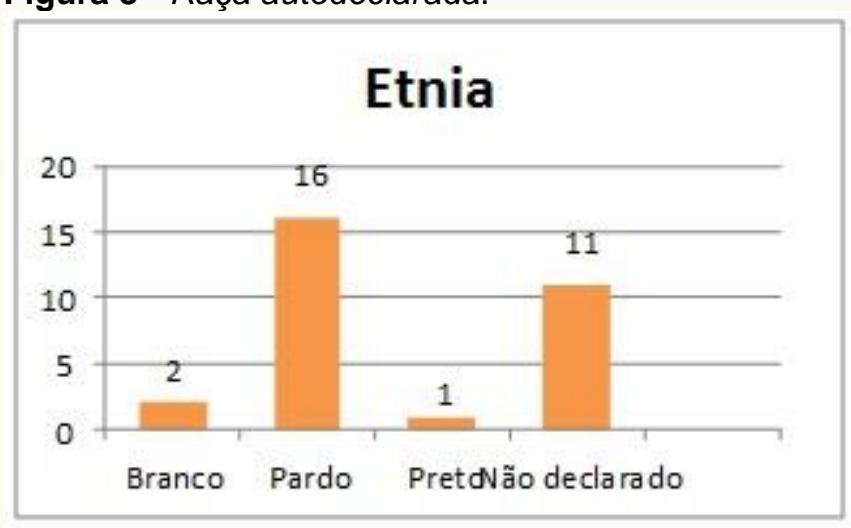

Nota-se, na Figura 3, que a maioria das mulheres - 53,3\% (16) se declararam pardas, e uma fração significativa não declarou sua cor - 36,7\% (11). Completando, 6,7\% (2) consideraram-se brancas e $3,3 \%$ (1) preta. No estudo realizado na zona urbana de Pelotas, houve predomínio de $83,2 \%$ de mulheres declaradas brancas [10].

Figura 4 - Localidade.

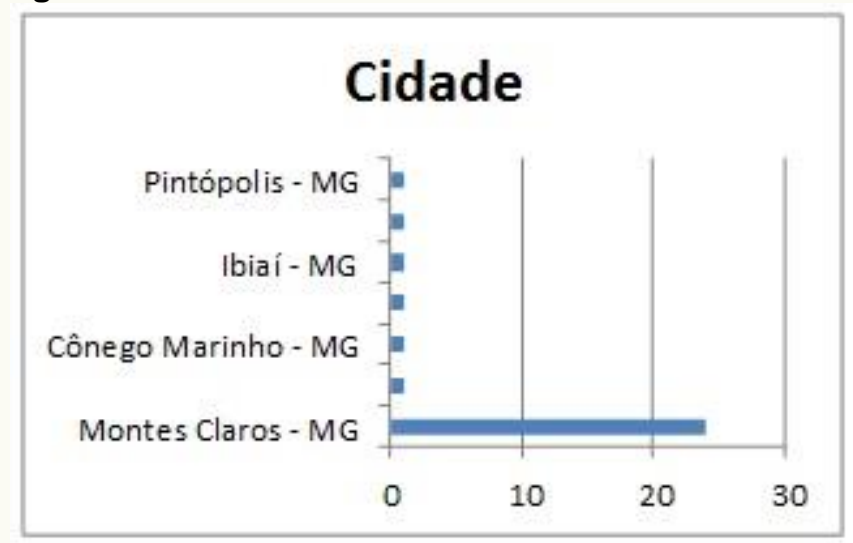

Sabe-se que o hospital universitário, espaço da investigação, atende a população do Norte de Minas, Vale do Jequitinhonha e Mucuri, Noroeste de Minas até o Sul da Bahia. Das mulheres atendidas, vinte e nove moram no Norte de Minas, destas $80 \%$ (24) residem em Montes Claros - cidade em que fica localizada a instituição; e apenas uma mulher é de outra região.

\section{Descrição dos CID e doenças prevalentes nas mulheres}

As doenças do CID são organizadas por categorias, isto é, códigos de três caracteres (uma letra e dois algarismos) e subcategorias (nesse caso, quando a uma categoria é atribuído um outro número, tem-se, portanto, as subdivisões de três caracteres iniciais acrescidos de um ponto e de outro número. Um conjunto de categorias que contém doenças semelhantes constitui um agrupamento [11].

A tabela I apresenta as doenças informadas nos prontuários analisados. Correspondendo a $13,4 \%$ (4) dos casos, a pneumonia foi a principal doença diagnosticada.

Como houve poucos casos de repetição, analisaram-se as doenças por categorias do CID, em que são agrupadas por doenças e natureza da lesão. As doenças do sistema gastrintestinal (doenças do fígado; e transtornos da vesícula biliar, das vias biliares e do pâncreas) totalizaram 20\% (6) dos casos; em seguida do sistema respiratório (influenza e pneumonia; e outras doenças da pleura) com 16,7\% (5) e do sistema circulatório (doenças das veias, dos vasos linfáticos e dos gânglios linfáticos, não classificadas em outra parte; doenças hipertensivas; e das artérias, das arteríolas e dos capilares) com 13,3\% (4) dos casos. 
Tabela I - Doenças e CID dos atendimentos.

\begin{tabular}{lccc}
\hline Doença & CID 10 & Quantidade & $\%$ \\
\hline Pneumonia & $\mathrm{J} 18$ & 4 & 13,4 \\
Trombose venosa profunda & $\mathrm{I} 82$ & 2 & 6,8 \\
Artrite & $\mathrm{M} 13$ & 2 & 6,8 \\
Colecistite aguda & $\mathrm{K} 81.0$ & 2 & 6,8 \\
HIV & $\mathrm{B} 24$ & 2 & 6,8 \\
Doença de Chagas & $\mathrm{B} 57$ & 1 & 3,3 \\
Crise hipertensiva & $\mathrm{I} 15$ & 1 & 3,3 \\
Colelitiase & $\mathrm{K} 80.2$ & 1 & 3,3 \\
Infecçáo estafilocócica & $\mathrm{A} 49.0$ & 1 & 3,3 \\
Abscesso cutâneo & $\mathrm{L} 02$ & 1 & 3,3 \\
Piodermite & L08.0 & 1 & 3,3 \\
Hepatite autoimune & $\mathrm{K} 75.4$ & 1 & 3,3 \\
Anemia fal ciforme & $\mathrm{D} 57.1$ & 1 & 3,3 \\
Acidente crotálico & $\mathrm{X} 20$ & 1 & 3,3 \\
Cirrose hepática aguda & $\mathrm{K} 70.3$ & 1 & 3,3 \\
Derrame pleural & $\mathrm{J} 90$ & 1 & 3,3 \\
Pancreatite aguda & $\mathrm{K} 85$ & 1 & 3,3 \\
Epistaxe & $\mathrm{R} 04.0$ & 1 & 3,3 \\
Doen ça vascular periférica & $\mathrm{I} .3$ & 1 & 3,3 \\
Celulite de face & $\mathrm{L} 03.2$ & 1 & 3,3 \\
Acidente botrópico & $\mathrm{X} 20$ & 1 & 3,3 \\
Hemoptise & $\mathrm{R} 04.2$ & 1 & 3,3 \\
Esclerose lateral amiotrófica & $\mathrm{G} 12.2$ & 1 & 3,3 \\
\hline
\end{tabular}

Em estudo cujo objetivo foi analisar as tendências das principais causas de internações hospitalares no Brasil, por faixa etária e região, no período de 1999 a 2006, constatou-se que, em menores de vinte anos, a primeira causa de internação foram as gastroenterites infecciosas e complicações (60,5 internações por 10 mil em 1999 e 53,3 por 10 mil em 2006); a segunda, asma (33,1 por 10 mil em 1999 e 22,6 por 10 mil em 2006); e, a terceira, as pneumonias bacterianas (7,4 internações por 10 mil em 1999 e 18,0 por 10 mil em 2006) [12].

Outro estudo realizado no hospital regional do Guará, região administrativa do Distrito Federal, mostrou que as principais causas presumidas das internações foram as doenças do aparelho respiratório, circulatório, geniturinário e as do sistema digestivo, representando juntas $75,9 \%$ destas causas naquele hospital [13].

Já em um hospital municipal em Barra das Garças, na Amazônia Legal, os diagnósticos que causaram maiores motivos de internação foram lesões, envenenamentos e algumas outras consequências de causas externas $(39,8 \%)$, seguido por doenças do aparelho digestivo com $25,3 \%$, doenças do aparelho circulatório $(6,0 \%)$ e doenças infecciosas e parasitárias $(5,5 \%)$, e as doenças respiratórias representaram apenas $4,3 \%$ dos casos [5].

\section{Comorbidades apresentadas e fatores associados à internação das mulheres}

O termo comorbidade se refere à presença de qualquer doença coexistindo adicionalmente, em um mesmo indivíduo, com uma doença particular. A presença desta doença adicional pode alterar o curso clínico, o período quando a doença é detectada, o prognóstico inicial, a eleição da terapêutica preferencial e os cuidados após o tratamento [14].

Os dados referentes às comorbidades foram coletados do item "história pregressa" da anamnese no prontuário clínico de cada mulher, 50\% (15) dessas mulheres não apresentava nenhuma comorbidade. A principal patologia associada encontrada foi hipertensão arterial, correspondendo a 30\% (9); seguida pelo diabetes mellitus - 13,3\% (4), como mostra a Figura 5. 
Figura 5 - Comorbidades associadas.

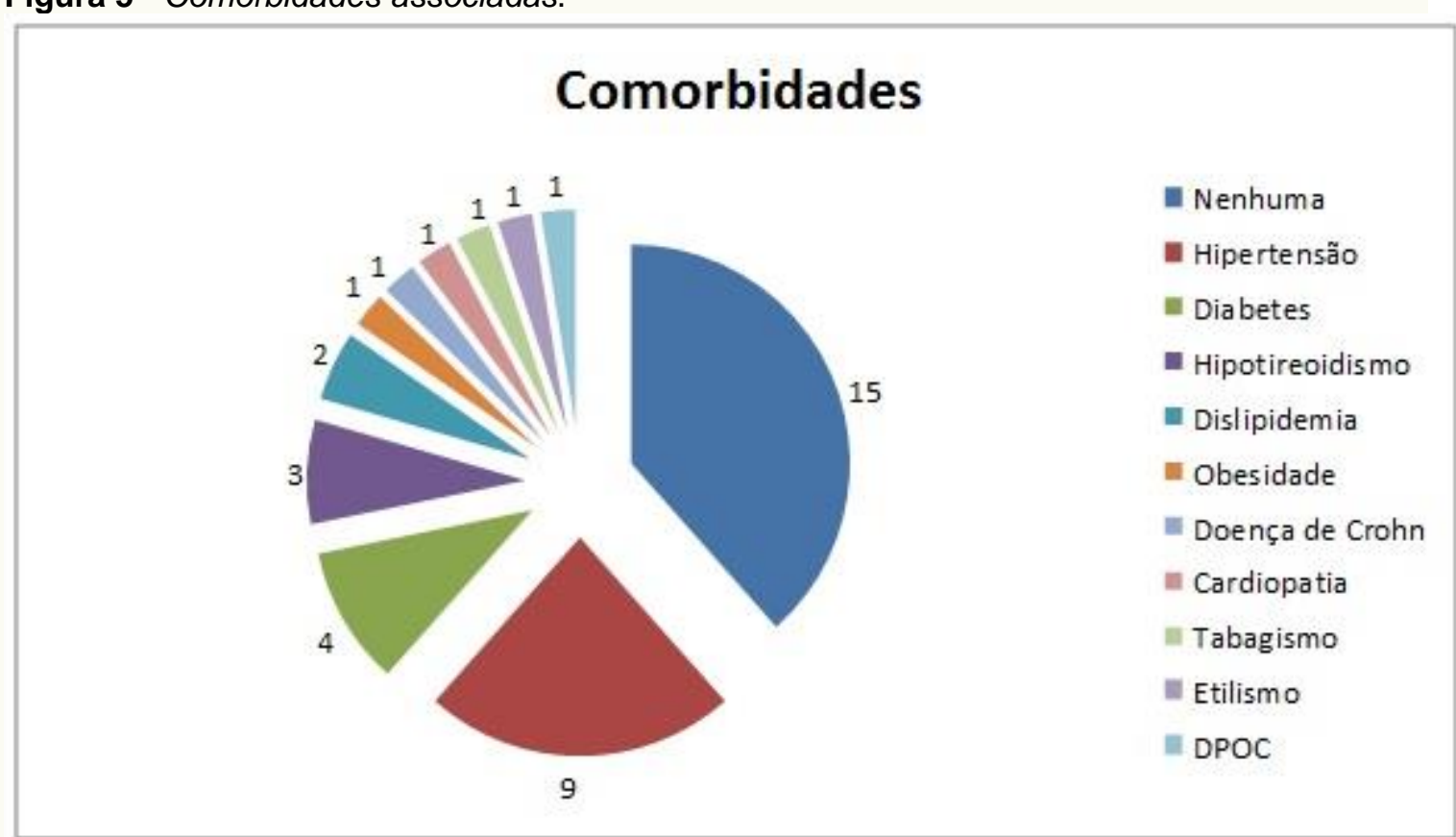

Estudo realizado em Fortaleza enfatizou as comorbidades associadas, com predomínio de 50 pacientes com insuficiência/infecção respiratória, 48 com hipertensão arterial sistêmica, 42 com septicemia, 35 com diabetes mellitus e 30 portadores da Síndrome da Imunodeficiência Adquirida (SIDA) [15].

Uma das finalidades de caracterizar as internações hospitalares é a possibilidade de identificar fatores associados à maior frequência de hospitalizações, a qual está relacionada em geral à maior gravidade e riscos à saúde. A identificação de fatores associados potencialmente modificáveis permite calcular o impacto de intervenções de cada fator na redução das internações hospitalares [10].

\section{Conclusão}

Os achados deste estudo demonstram que a maioria das mulheres é adulta e solteira. A principal patologia encontrada foi pneumonia, embora as doenças do sistema gastrintestinal aparecessem em maior número. Metade das mulheres não apresentava doença associada, enquanto a principal comorbidade foi hipertensão arterial.

Os bancos de dados e as produções bibliográficas referentes à temática não possuem ricas informações sobre comorbidades, o que contribuiria para analisar a utilização dos serviços de saúde e a identificação dos principais fatores que causam maior frequência de internações de mulheres.

Sabendo-se sobre os principais acometimentos em mulheres internadas, a implementaçãode políticas de prevenção, proteção e promoção à saúde na atenção primária objetiva a redução de ocorrência de internações, assim como envolve a equipe hospitalar de modo cooperativo e participativo em pesquisas e contribui para o aperfeiçoamento do cuidado e melhoria na qualidade de atendimento específico.

Portanto, tais achados somam às demais publicações existentes para utilização das informações, considerando que se cumpra o objetivo de devolvê-las à comunidade e profissionais envolvidos, e aprofunde os estudos sobre os determinantes das internações hospitalares de mulheres. Além disso, torna-se evidente a necessidade de profissionais qualificados para o atendimento deste público. 
1. Instituto Brasileiro de Geografia e Estatística (IBGE). Censo Demográfico 2010. [citado 2013 Nov 25]. Disponível em URL: http://www.ibge.gov.br/home/

2. Ministério da Saúde. Departamento de Informática do Sistema Único de Saúde DATASUS 2012. [citado 2013 Nov 25]. Disponível em URL http://cidades.ibge.gov.br/painel/saude.php?

3. Batista LE, Almeida MAS, Morita I,Volochko A, Rea MF. De que adoecem e morrem as mulheres em São Paulo. Saúde e Sociedade 2000;9(1/2):17-47.

4. Toledo AD. Acurácia de enfermeiros na classificação de risco em unidade de pronto socorro de um hospital municipal de Belo Horizonte [Tese]. Belo Horizonte, MG: Escola de Enfermagem /UFMG; 2009. 138 p.

5. Trindade NR, Neto MAS, Toledo OR, Moraes EV, Ferrari CKB, David FL. Causas de internação hospitalar em adultos em um município da Amazônia Legal, Brasil. J Manag Prim Health Care 2013;4(2):70-6.

6. Freitas P. Triagem no serviço de urgência. 2a ed. Manchester: Grupo de Triagem de Manchester; 2002.

7. Fontelles MJ, Simões MG, Farias SH, Fontelles RGS. Metodologia da pesquisa científica: diretrizes para a elaboração de um protocolo de pesquisa. Rev Para Med 2009;23(3).

8. Guerra MO. Como fazer um projeto de pesquisa. 3a. ed. Juiz de Fora: EDUFJF; 1997. $49 \mathrm{p}$.

9. Minayo MC. O desafio do conhecimento: pesquisa qualitativa em saúde. 7a. ed. São Paulo: Hucitec-Abrasco; 2000. 270 p.

10. Costa JSD, Reis MC, Filho CVS, Linhares RS, Piccinini F, Fantinel E. Prevalência de internação hospitalar e fatores associados em Pelotas, RS. Rev Saúde Pública 2010;44(5):923-33.

11. 10 $0^{\mathrm{a}}$ Revisão da Classificação Internacional de Doenças. [citado 2013 Nov 25]. Disponível em URL: http://www.datasus.gov.br/cid10/V2008/apresent.htm

12. Moura BLA, Cunha RC, Aquino R, Medina MG, Mota ELA, Macinko J et al. Principais causas de internação por condições sensíveis à atenção primária no Brasil: uma análise por faixa etária e região. Rev Bras Saúde Matern Infant 2010;10(Supl1):S83S91.

13. Oliveira WL, Branco AB. Avaliação da antibioticoterapia em pacientes internados no hospital regional do Guará-DF. Comunic Ciênc Saúde 2007;18(2):107-14.

14. Zilberman ML, Giusti JS. A mulher e a dependência química: uma perspectiva feminina. In: Gigliotti A, Guimarães A, eds. Dependência, Compulsão e Impulsividade. Rio de Janeiro: Rubio; 2007. p.19-29.

15. Oliveira FC, Alves MDS, Bezerra AP. Comorbidades e mortalidade de pacientes com doença renal: atendimento terceirizado de nefrologia. Acta Paul Enferm 2009;22 (Especial-Nefrologia):476-80. 